\title{
Discarded old antibiotics as a new arsenal for multi- resistant isolates in diabetic foot ulcer infections: Therapeutic value of parenteral Colistin versus multi-resistant Pseudomonas speciesisolate strains. Case Report
}

\author{
Ahmed Shabhay ( $\sim$ ahmedshabio84@gmail.com ) \\ Institute of Infectious Diseases and Research, Lugalo Military College of Medical Sciences (MCMS) and \\ General Military Hospital (GMH), P.O. Box 60000 Dar es Salaam, Tanzania https://orcid.org/0000-0001- \\ 9214-5415
}

Jaffu O. Chilongola

Kilimanjaro Clinical Research Institute (KCRI) P.O. Box 2236 Moshi https://orcid.org/0000-0003-29976747

\section{Stephen Mshana}

Department of Microbiology and Immunology, Catholic University of Health and Allied Sciences (CUHAS), P.O. Box 1464 Mwanza, Tanzania https://orcid.org/0000-0002-7526-6271

\section{Zarina Shabhay}

Muhimbili Orthopedic Institute P.O. Box 65474 Dar es Salaam https://orcid.org/0000-0002-1484-3338

\section{Jeff Van Baal}

ZGT Academy, Hospital Group Twente, Almelo/Hengelo, The Netherlands

\section{Anande Salewi}

Department of General Surgery, Kilimanjaro Christian Medical Centre, P.O. Box 3010 Moshi, Tanzania

Theresia Mwakyembe

Department of General Surgery, Kilimanjaro Christian Medical Centre, P.O. Box 3010 Moshi, Tanzania

\section{Kondo Chilonga}

Department of General Surgery, Kilimanjaro Christian Medical Centre, P.O. Box 3010 Moshi, Tanzania

\section{David Msuya}

Department of General Surgery, Kilimanjaro Christian Medical Centre, P.O. Box 3010 Moshi, Tanzania https://orcid.org/0000-0003-1653-3365

\section{Samwel Chugulu}

Department of General Surgery, Kilimanjaro Christian Medical Centre, P.O. Box 3010 Moshi, Tanzania Pius Horumpende

Institute of Infectious Diseases and Research, Lugalo Military College of Medical Sciences (MCMS) and General Military Hospital (GMH), P.O. Box 60000 Dar es Salaam, Tanzania https://orcid.org/0000-0003- 


\section{Case Report}

Keywords: Colistin, Pseudomonas aeruginosa, amputation, case report

Posted Date: September 2nd, 2021

DOI: https://doi.org/10.21203/rs.3.rs-702608/v1

License: (c) (i) This work is licensed under a Creative Commons Attribution 4.0 International License. Read Full License

Version of Record: A version of this preprint was published at International Journal of Basic \& Clinical Pharmacology on November 22nd, 2021. See the published version at https://doi.org/10.18203/23192003.ijbcp20214509. 


\section{Abstract \\ Background}

Antimicrobial resistance to current novel antibiotics is posing a major threat to both human and zoonotic life. This poses a serious potential of rolling back to pre-antibiotic era clinical settings. Antimicrobial discovery pipeline has dried up as major pharmaceutical brands have shifted to long term chronic illness drugs production. In the absence of new novel antibiotics molecules, clinicians have resorted into a desperate last resort to review and re-introduce prior discarded antibiotics as their new weaponry in the fight against multi-resistant Gram-negative bacteria.

\section{Case presentation:}

We report a case of a 77 years old bed ridden diabetic and hypertensive with renal impairment diagnosed with bilateral lower limbs wet gangrene. She underwent transfemoral on her right and transtibial amputation on her left lower limb. She developed Surgical site infection on her right stump and wet gangrene on her left stump. Surgical toilet, debridement and stump revision was done on her right stump and a transfemoral amputation on her left lower limb. Pus swab on her right stump revealed carbapenem resistant strains of Pseudomonas aeruginosa. She was instituted on parenteral colistin and showed no bacterial growth 7 days post treatment. She suffered an ischaemic cerebral vaso-occlusive stroke during her hospital stay. CT angiography revealed distal infra-renal abdominal aorta multiple calcified plaques, multiple calcified plaques and completely obstructing thrombus both on common and external iliac arteries, seen downstream to both superficial femoral arteries with multiple collaterals in both thighs. The included portion of the lower lung fields showed a large filling defect in the right main pulmonary artery extending to the lower lobe branches suggestive of right-sided pulmonary embolism. She developed hypostatic pneumonia, bed sores and her condition deteriorated and she unfortunately succumbed from her comorbidities. Despite our patient succumbing to her multiple co-morbidities we report this case to highlight the isolation of carbapenem resistant strains of Pseudomonas aeruginosa and the efficacy and safety of colistin as a salvage antibiotic in renal impaired patients.

\section{Conclusions}

Colistin can be safely used as a last reserve antibiotic for multi-resistant strains of Pseudomonas aeruginosa infection even in patients with renal impairment.

\section{Background}

A major breakthrough in the history of modern medicine was the discovery of antibiotics(1). However due to irrational practice of antibiotics usage, pathogens have developed and shared resistanceto commonly prescribed current and active antibiotics(2). In the current era of increasing Antimicrobial resistance 
(AMR)to current novel antibiotics which is a major threat to human life, discovery and development of new novel antibiotics has greatly decreased(3), due to pharmaceutical corporations losing interest in the arena of antimicrobial manufacture thus driving clinicians back to old discarded narrow therapeutic window antibiotic molecules(3) such as the nephrotoxic colistin(4)(5), an old class of cyclic polypeptide antibiotic that was discovered in 1947 from Paenibacilluspolymyxa(6)to combat carbapenemresistantGram-negative bacteria such as Pseudomonas aeruginosa(1)(2)(5)(7)(8)(9).Carbapenems have been the last resort in Extended Spectrum of Beta Lactamase producing Pseudomonas aeruginosa strains but of recent microbiological studies globally, and as it is in our case study, resistant strains have been isolated, with colistin remaining the only viable last desperate resort despite its nephrotoxicity potentiality(5). Morbidity and mortality due to multi-resistant nosocomial Gram negative bacterial infections is high(2)(5).Multi-drug resistant $P$ seudomonas aeruginosa strains are increasingly being isolated from patients globally(5). In this case report we set out to show the challenges of management of a multi-resistant Pseudomonas aeruginosa strain in a diagnosed chronic kidney diseased diabetic and hypertensive patient with bilateral lower limbs wet gangrene. Our patient despite her co morbidity of chronic kidney disease tolerated well the nephrotoxic antibiotic molecule colistin. This encourages the reintroduction of colistin in carbapenem resistant bacterial strains in diabetic patients with renal comorbidity.

\section{Case Presentation}

A 77 years old bed ridden female, with history of cerebral vascular accident 6 years prior admission, diagnosed diabetic, chronic kidney disease stage one, and hypertensive on oral hypoglycemics and antihypertensives diagnosed with bilateral lower limbs wet gangrene extending from the foot up to proximal one third tibia on the right limb (Fig. 1(a \& B)) and limited on the left foot, was admitted at our General Surgical ward in KCMC Hospital. Her Glasgow coma scale was 12/15(E/4, V/3, M/6).Muscle power was $4 / 5$ on both upper and lower limbs bilaterally. Her vitals on admission were: (BP

$131 / 69 \mathrm{mmHg}$, R.R 22cycles/min, Pulse rate 82 beats/min, body temperature $35.4^{\circ} \mathrm{C}$ and Oxygen saturation $93 \%$ on Room Air. On admission she was on parenteral ceftriaxone, cloxacillin, frusemide with oral atorvastatin, amlodipine and glibenclamide. Her Haemoglobin level was $10.3 \mathrm{~g} / \mathrm{dL}$, Leucocyte count $25.69 \times 10^{9} / \mathrm{L}$ with neutrophilia of $23.69 \times 10^{9} / \mathrm{L}$, platelets $109 \times 10^{9} / \mathrm{L}$, Serum creatinine level $109 \mu \mathrm{mol} / \mathrm{L}$, Urea $14.55 \mathrm{mmol} / \mathrm{L}$ and random Blood Glucose was $10 \mathrm{mmol} / \mathrm{L}$. ECG revealed atrial fibrillation with ST elevation.

She was scheduled for emergency right transfemoral and left transtibial amputations. Post-operative she was instituted on parenteral merogram. She developed surgical site infection on her right above knee amputated stump (Fig. 2) and wet gangrene on her left transtibial stump (Fig. 3). Blood culture revealed Citrobacter species sensitive to amoxylin/clavulanic acid, amikacin, cefotaxime and gentamicin with resistance to ampicillin and ceftazidime. Pus swab on right AKA stump reveledPseudomonas species resistant to amikacin, ampicillin, ceftriaxone, ceftazidime, cefotaxime, gentamicin, imipenem and piperacillin (Fig. 4). Pus swab left BKA stump reveled Staphylococcus species and (Pseudomonas 
species) resistant to cefoxitin, erythromycin and imipenem with sensitivity to clindamicin and gentamicin. CT angiography revealed distal infra-renal abdominal aorta multiple calcified plaques and completely obstructing thrombus both on common and external iliac arteries, seen downstream to both superficial femoral arteries with multiple collaterals in both thighs. The included portion of the lower lung fields showed a large filling defect in the right main pulmonary artery extending to thelower lobe branches suggestive of right-sided pulmonary embolism.

She was scheduled for right AKA stump surgical toilet, debridement and revision and left mid-third transfemoral amputation. She was then instituted on parenteral colomycin ${ }^{\circledR} 150 \mathrm{mg}$ bd for 7 days, low molecular weight heparin, soluble aspirin, clopidogrel, analgesia, oral hypoglycemics and antihypertensives. Her blood glucose levels were maintained below $10 \mathrm{mmol} / \mathrm{L}$. Her serial creatinine and glomerular filtration rate post colomycin institution were $(114 \mu \mathrm{mol} / \mathrm{L}, 44 \mathrm{ml} / \mathrm{min})(86 \mu \mathrm{mol} / \mathrm{L}, 59 \mathrm{ml} / \mathrm{min})$ and $(62 \mu \mathrm{mol} / \mathrm{L}, 89 \mathrm{ml} / \mathrm{min})$ respectively. She had episodes of fever spikes reaching $40^{\circ} \mathrm{C}$ which were controlled by paracetamol. A control blood culture was done 7 days post institution of colomycin and revealed no growth after 5 days incubation. Our patient sustained a Transient IschaemicAttack (TIA), developed hypostatic pneumonia, bed sores and her condition deteriorated and she unfortunately succumbed from her comorbidities.

\section{Discussion And Conclusion}

The worldwide prevalence of multidrug-resistant Gram-negative bacteria in particular Pseudomonas aeruginosa, Acinetobacterbaumanniiand Klebsiella pneumonia is escalating at an alarming rate(1)(3) with polymyxins being the only active antibiotics(6). In the Unites States of America, up to $16 \%$ of Pseudomonas aeruginosa isolates in the time period between 1997 and 2000 were resistant to at least three principal anti-pseudomonas medications(7). Pseudomonas aeruginosa has proved to be resistant to a number of antibiotics, including aminoglycosides, quinolones and $\beta$-lactams $(10)$. The absence of new antibiotics against resistant bacteria due to the decline of the antibiotic discovery pipeline, has led to renewed interest in reviving the use of older antibiotics that were considered too toxic for clinical use, in particularpolymyxins ( $B$ and $E$ ), to be the only salvage arsenal in the antibiotics armory in multi-resistant gram negative bacterial infections in particular Pseudomonas aeruginosa(1).

$P$. aeruginosa is a perilous and feared ubiquitous Gram-negative, opportunistic nosocomial acquired bacterium(5), responsible for $10 \%$ of all hospital-acquired infections(11), in immunocompromised hosts, culprit in ventilator-associated pneumonia, resistant to anti-microbials due to its ability to produce a variety of toxins, resisting phagocytosis, encoding large percentage of controlling enzymes important for metabolism and transportation with its high level of intrinsic resistance to most anti-microbials through restricted outer membrane permeability, efflux systems that pump anti-microbials out of the cell and production of antibiotic-inactivating enzymes such as $\beta$-lactamases giving it great metabolic versality and high adaptability to resist ecological changes thus its ability to colonize a wide variety of settings (12)(10). It is mostly isolated from patients with burns injuries, cystic fibrosis, and neutropenia(5)(12). 
The use of old antibiotics resembles the antibiotic rotation scheme where some antibiotics are intentionally withheld from use to reduce chances of developing resistance to certain pathogens(7). Old antibiotics have a major advantage to the currently used antibiotics by the fact that their use has been greatly limited in recent years(7). It's well known that emergence of resistance to antimicrobials is from the misuse of the antimicrobial itself(7). Challenge posed by many old discarded antibiotics is their toxicity and adverse effects, however their efficacy can be improved by chemically modifying their compounds to enable better tissue penetration and extend half-livestherefor increasing potency to certain pathogens.(7).

Colistin (polymyxin E) is a multicomponent polypeptide antibiotic comprised mainly of colistin A and Bantibiotic discovered in 1949(3) and has been available since 1959 for the treatment of infectious diseases caused by Gram-negative bacteria used throughout in the 1960s and 1970s, discarded due to reports of nephrotoxicity for the potentially less-toxic aminoglycosides(1)(6)(7)(4). The clinical picture of colistin nephrotoxicity include a decrease in clearance of creatinine, proteinuria, cylindruria or oliguria(4).This led to decline in its use from the 1970 s to the early 2000 s(6). However it has been observed that the nephrotoxicity is dose dependent, usually but not always reversible and end organ kidney damage rarely reported(4).

After the emergence of multi-resistant Gram negative bacteria, its re-use re-emerged with fewer reported side effects compared to aminoglycosides which had replaced it more than 3 decades ago(7). The toxicity previous reported could be owed to higher colistin doses administered, the administration of the more toxic form of colistin sulphate or the co-administration of nephrotoxic drugs(7).

However a concerning and scary reality exist as there have been studies were isolates showed resistance to both colistin and carbapenems(2). Colistin resistance have been reported in UK, were resistant strains of P.aeruginosain cystic fibrosis have been observed, colistin resistant carbapenemase-producing K.Pneumoniae,resistant strains of A.baumannii and polymyxin resistant Escherichia coli(2). A study in India identified resistant strains of Escherinchia coli, K.pneumoniae, P.aeruginosa, and Acinetobacterbaumannito bothmeropenem and colistin(2). These are frightening reports to clinicians who face multi-resistant strains of Gram-negative bacteria, with colistin as there only single salvage antibiotic losing its potency. However the advent of colistin resistance has been described in high-use settings(9).

A new hope exists as colistin can be used with combination with rifampicin or sulbactam antibiotics to increase efficacy for the treatment of multi-drug resistant Gram negative bacteria(1)(6)(8)despite the increased risk in toxicity(8).Animal studies have shown combination of colistin with either rifampicin, carbenicillin, piperacillin and imipenem for treatment of P.aeruginosa, A.baumannii or E. coli infections there were significant reduced mortality rates(8).However, there is a need for clinicians to share their experience on the efficacy and safety of single therapy colistin use versus colistin combination therapy.Best approach to this is randomized controlled trials comparing and contrasting effectiveness and safety of colistin monotherapy versus colistin combination therapy(8). 
A new surge of antibiotic discovery research is needed in these desperate times of increasing AMR. Contrary to this need, nearly half of all major antimicrobial producers in Japan and USA have halted or decreased antimicrobial production almost 30 decades ago(3)(7)(13). The absence of alternative antibiotics to treat the ever increasing rate of AMR among even common bacteria is likely to end up in a clinical scenario where colistin will be the only last line salvage therapeutic drug against multidrugresistant Gram-negative pathogens in the 21 st century(6).In clinical scenarios where $\beta$-lactam, aminoglycoside or quinolone is rendered ineffective, the polymyxins, especially colistin, serve as the final alternative arsenal(6).

It is understood that resistance is acquired through a number of mechanisms including selection pressure mainly due to miss use of the drugs. It is an open issue whether restricting antimicrobial usage contributes to the elimination of multidrug-resistant bacteria. Resistance mutations frequently have associated fitness costs. However, such costs may decline subsequently through the accumulation of compensatory mutations(14). It has previously been demonstrated that drug-resistance declines in an antibiotic-free laboratory environment in as few as 480 generations(15) where $64.7 \%$ of drug-resistant $E$. coli strains showed elevated susceptibilities to at least one antibiotic investigated. Reductions in antimicrobial prescription have been reported to achieve specific, localized decreases in antimicrobial resistance in many parts of the world(16). Although antibiotic withdrawal has not been a formal strategy for managing AMR, our finding underscores the possibility of antibiotic withdrawal strategy as a promising approach to contain AMR.

In this case, our patient was a chronic kidney diseased diabetic and hypertensive. She developed carbapenem resistant Pseudomonas aeruginosa diabetic foot ulcer infection. She was instituted on the nephrotoxic antibiotic molecule colistin. Her renal blood work during treatment were monitored and were within normal acceptable ranges. This gives new hope for the safe and efficacious re-introduction of colistin even in renal comprised patients.

In this current clinical scenario of increasing AMR, old and current active antimicrobials should be engaged in combating severe infections due to multi-drug resistant strains of Gram-negative bacteria. Individual countries drugs and food agencies should review the old discarded antibiotics as their potential new armory in the fight against current resistant bacterial strains. However, this should be viewed as potential dangerous last step towards re-entering the same clinical scenario of the preantibiotic era were clinicians had virtually no antibiotic molecules to treat infections. Strict laws should be put to place to enforce the rational use of current active antibiotics.Colistin can be safely used as a last reserve antibiotic for multi-resistant strains of Pseudomonas aeruginosa infection even in patients with renal impairment. Dose adjustments are needed in patients with renal impairment. In severe multiresistant bacterial strains, it can be combined with rifampicin or a carbapenems to increase efficacy. Its use should be restricted only as a last resort to avoid re-emergence of colistin resistance.

\section{Declarations}




\section{Ethics approval and consent to participate}

This case report was conducted according to principles and ethical standards set by the Kilimanjaro Christian Medical University College Research and Ethical Committee (CREC). The case report was conducted according to the ethical principles for medical research in accordance to the Declaration of Helsinki. Confidentiality was ensured in that no personal identifying information was revealed. The Patient and her family were clearly made to understand that not participating in the study would in no way jeopardize clinical management in the ward. Written informed consent was obtained from the patient's son for writing of this case report; additionally, accompanying images have been censored to ensure that the patient cannot be identified. A copy of the consent is available on record. Approval to publish was obtained from relevant authorities.

\section{Consent for publication}

Written informed consent was obtained from the patient for publication of this case report and any accompanying images. A copy of the written consent is available for review by the Editor-in-Chief of this journal.

\section{Availability of data and materials}

All data and materials pertaining to this case report can be made available on request.

\section{Competing interest}

The authors declare they have no competing interests. All authors of the manuscript have read and agreed to its contents.

\section{Funding}

No funding was obtained for this case report.

\section{Authors' contributions}

All authors AS, JF, SM, ZS, JVB, AS, TM, KC, DM, SM and PH have read and approved the final version of the manuscript. All authors made a significant contribution to the work reported, whether that is in the conception, study design, execution, acquisition of data and interpretation, or in all these areas; took part in drafting, revising or critically reviewing the article; gave final approval of the version to be published; have agreed on the journal to which the article has been submitted; and agree to be accountable for all aspects of the work.

\section{Abbreviations}

AMR- Anti-microbial Resistance 
BKA- Below Knee Amputation

\section{Acknowledgement}

The authors would like to thank the patient for permission to share their medical history for educational purposes and publication.

\section{References}

1. Li J, Nation RL, Turnidge JD, Milne RW, Coulthard K, Rayner CR, et al. Colistin: the re-emerging antibiotic for multidrug-resistant Gram-negative bacterial infections. Vol. 6, Lancet Infectious Diseases. 2006. p. 589-601.

2. Ramesh N, Prasanth M, Ramkumar S, Suresh M, Tamhankar AJ, Gothandam KM, et al. Colistin susceptibility of gram-negative clinical isolates from Tamil Nadu, India. Asian Biomed. 2016;10(1):35-9.

3. Cassir N, Rolain JM, Brouqui P. A new strategy to fight antimicrobial resistance: The revival of old antibiotics. Front Microbiol. 2014;5(OCT):1-15.

4. Ordooei Javan A, Shokouhi S, Sahraei Z. A review on colistin nephrotoxicity. Eur J Clin Pharmacol. 2015;71(7):801-10.

5. Azimi L, Lari AR. Colistin-resistant Pseudomonas aeruginosa clinical strains with defective biofilm formation. GMS Hyg Infect Control. 2019;14:Doc12.

6. Biswas S, Brunel JM, Dubus JC, Reynaud-Gaubert M, Rolain JM. Colistin: An update on the antibiotic of the 21st century [Internet]. Vol. 10, Expert Review of Anti-Infective Therapy. 2012 [cited 2020 Nov 9]. p. 917-34. Available from: https://www.medscape.com/viewarticle/772588_2

7. Falagas ME, Grammatikos AP, Michalopoulos A. Potential of old-generation antibiotics to address current need for new antibiotics. Expert Rev Anti Infect Ther. 2008;6(5):593-600.

8. Petrosillo N, loannidou E, Falagas ME. Colistin monotherapy vs. combination therapy: Evidence from microbiological, animal and clinical studies. Clin Microbiol Infect [Internet]. 2008;14(9):816-27. Available from: http://dx.doi.org/10.1111/j.1469-0691.2008.02061.x

9. Yahav D, Farbman L, Leibovici L, Paul M. Colistin: New lessons on an old antibiotic. Clin Microbiol Infect [Internet]. 2012;18(1):18-29. Available from: http://dx.doi.org/10.1111/j.14690691.2011.03734.x

10. Pang Z, Raudonis R, Glick BR, Lin TJ, Cheng Z. Antibiotic resistance in Pseudomonas aeruginosa: mechanisms and alternative therapeutic strategies. Biotechnol Adv [Internet]. 2019;37(1):177-92. Available from: https://doi.org/10.1016/j.biotechadv.2018.11.013

11. Aloush V, Navon-Venezia S, Seigman-Igra Y, Cabili S, Carmeli Y. Multidrug-resistant Pseudomonas aeruginosa: Risk factors and clinical impact. Antimicrob Agents Chemother. 2006;50(1):43-8.

12. Sivanmaliappan TS, Sevanan M. Antimicrobial susceptibility patterns of pseudomonas aeruginosa from diabetes patients with foot ulcers. Int J Microbiol. 2011;2011. 
13. Jackson N, Czaplewski L, Piddock LJV. Discovery and development of new antibacterial drugs: Learning from experience? J Antimicrob Chemother. 2018;73(6):1452-9.

14. Mwanza S, Joshi S, Nambozi M, Chileshe J, Malunga P, Kabuya JBB, et al. The return of chloroquinesusceptible Plasmodium falciparum malaria in Zambia. Malar J. 2016;15(1):1-6.

15. Dunai A, Spohn R, Farkas Z, Lázár V, Györkei, Apjok G, et al. Rapid decline of bacterial drug-resistance in an antibiotic-free environment through phenotypic reversion. Elife. 2019;8:1-20.

16. Barbosa TM, Levy SB. The impact of antibiotic use on resistance development and persistence. Drug Resist Updat. 2000;3(5):303-11.

\section{Figures}
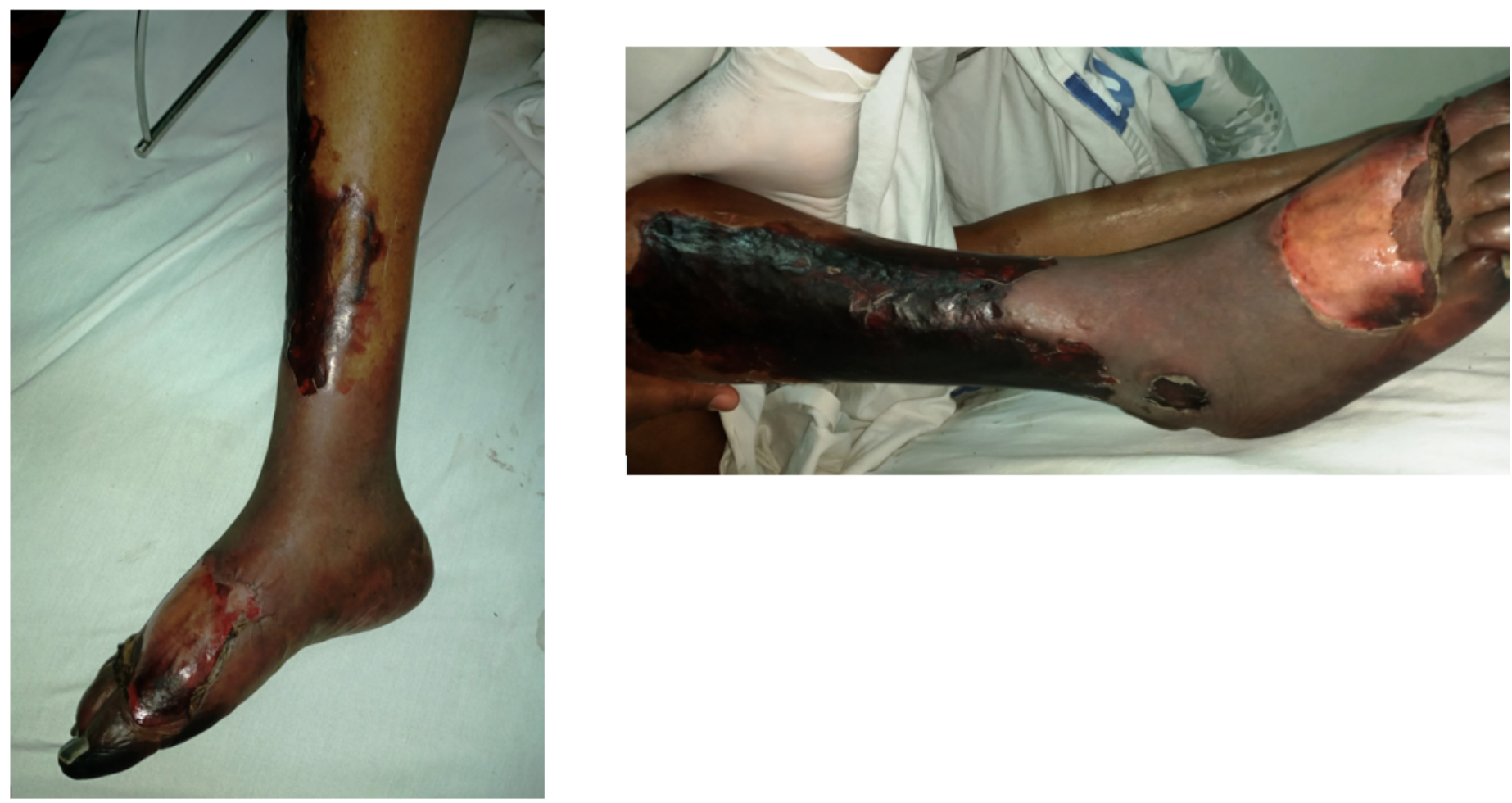

\section{Figure 1}

bilateral lower limbs wet gangrene extending from the foot up to proximal one third tibia on the right limb 


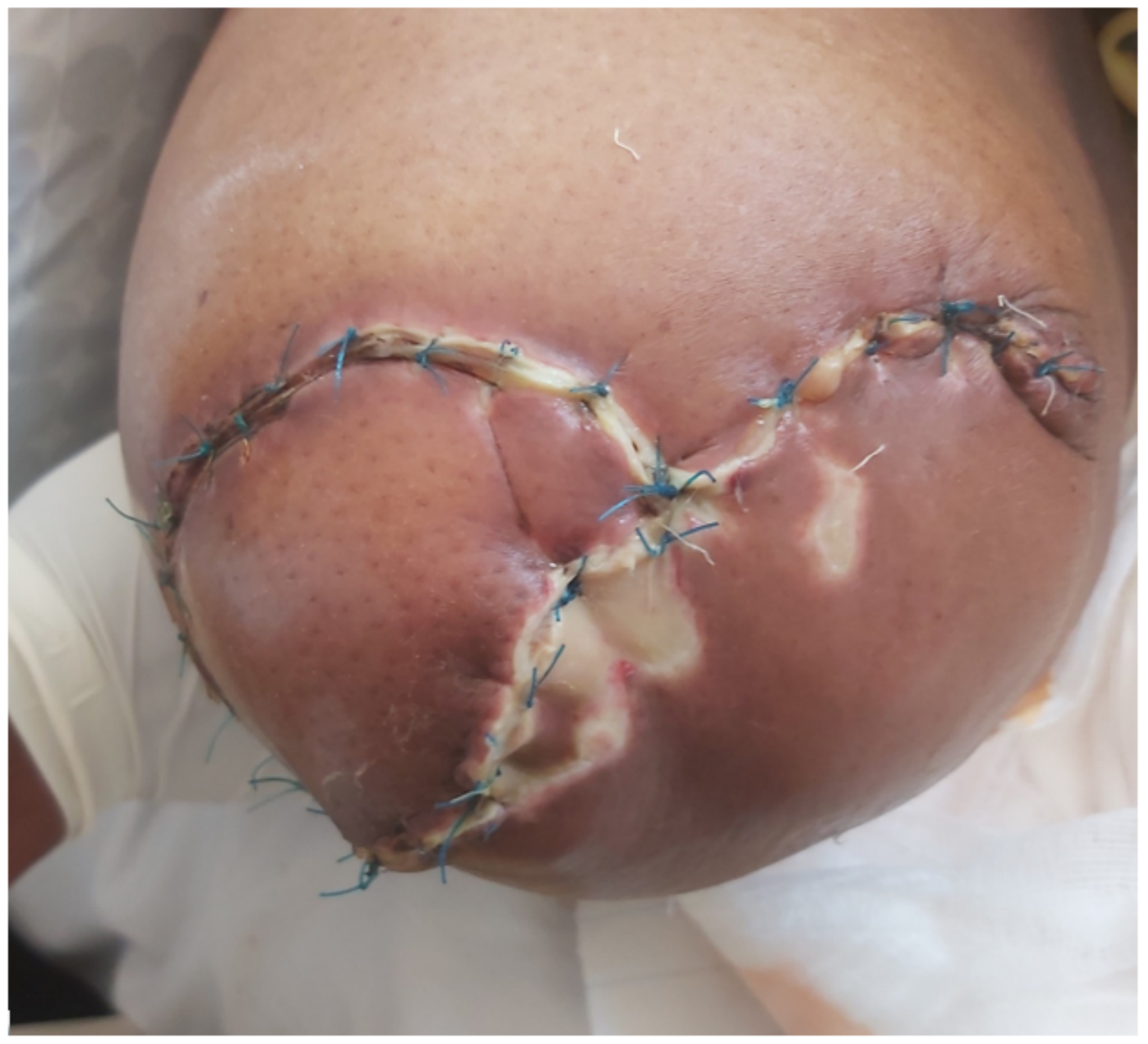

Figure 2

surgical site infection on her right above knee amputated stump 


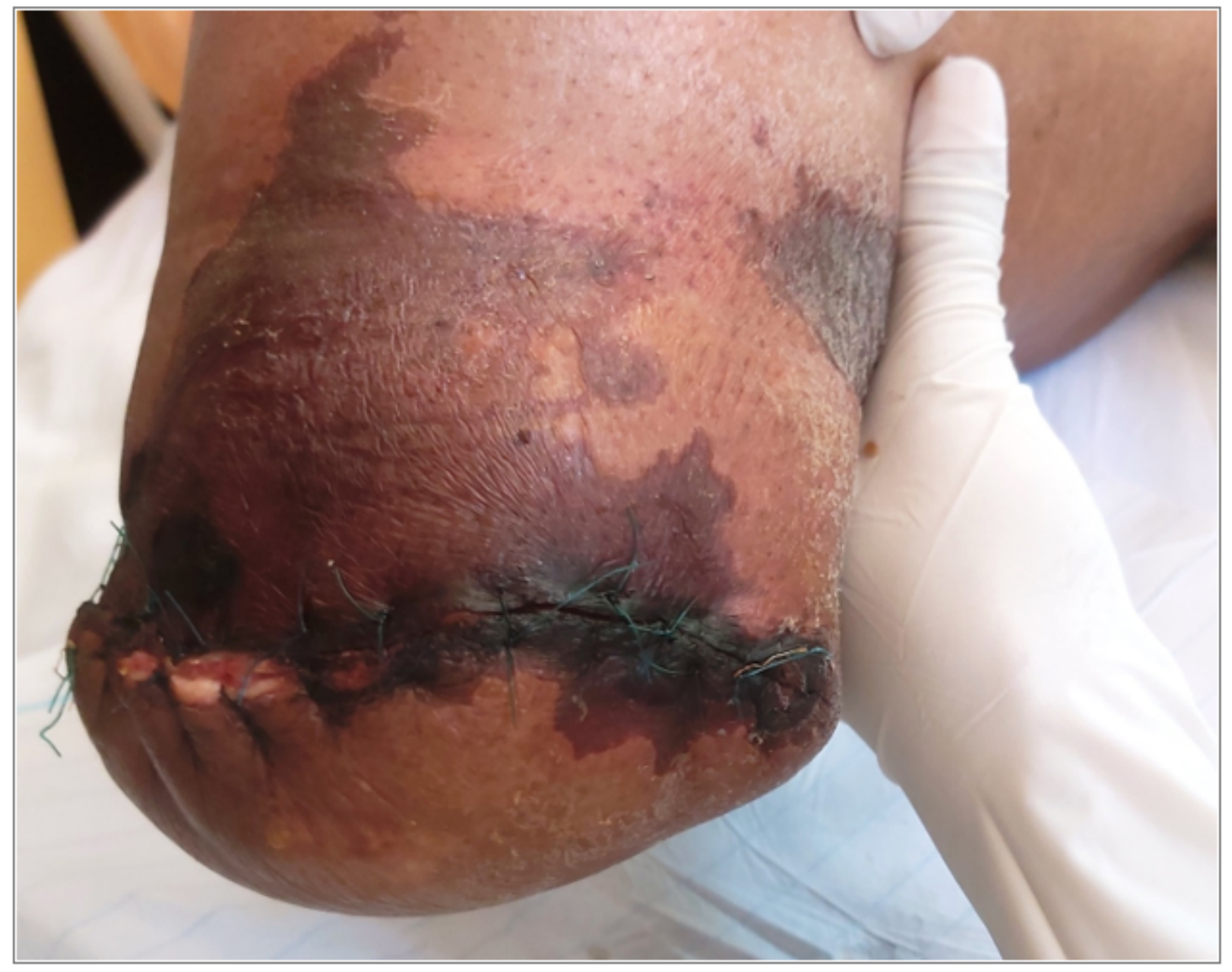

Figure 3

wet gangrene on her left transtibial stump 


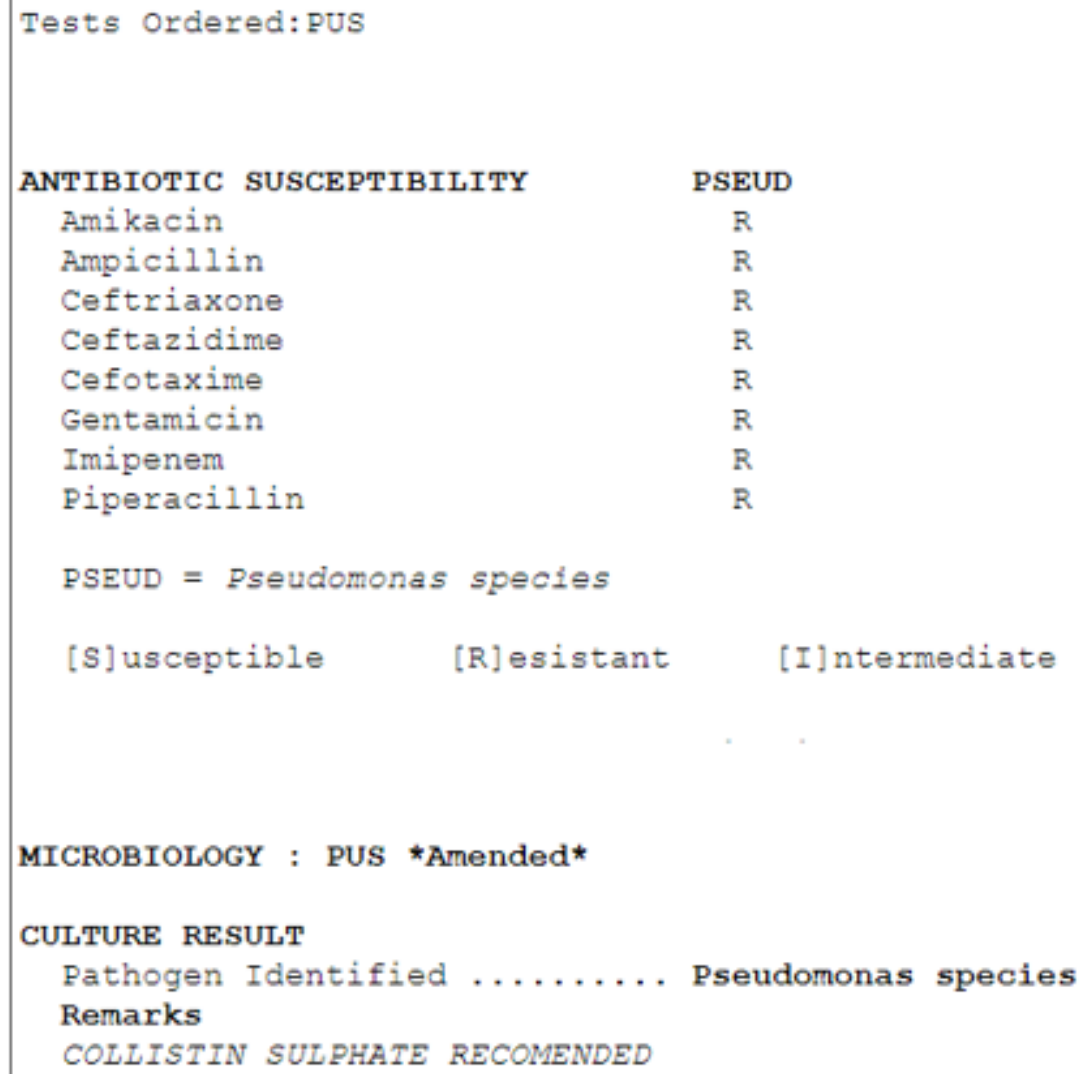

\section{Figure 4}

Pus swab on right AKA stump reveledPseudomonas species resistant to amikacin, ampicillin, ceftriaxone, ceftazidime, cefotaxime, gentamicin, imipenem and piperacillin 